\title{
A Multidisciplinary Approach to Problem Solving in Failure Analysis: What To Do When Microscopy Isn't Enough
}

\author{
Valerie P. Woodward
}

Noveon, Inc., Measurement Science, 9911 Brecksville Rd., Cleveland, OH, 44141

We all like to think that our own method(s) can answer most of our clients' questions. When a scientist, engineer, marketing associate, or business group leader comes into your service lab office in a panic, he or she is usually looking for the ultimate answer in less than an hour for free. As analysts, we know that we normally cannot meet the criteria presented to us, but we must try - in most cases, money and company credibility are on the line. We may also know that the problem will require more probing than microscopy and microanalysis can provide, and it is up to us to recommend other testing, educate the client on why it might be necessary, reasonably understand the other methods so that we can pull all the information together, and act as the advocate for the client when we bring the sample/problem to the other labs. Thus, the challenge is upon us.

An engineer brings a rubber blend part to the micro lab; the part began to leak in usage at very low temperatures, leading to a troublesome, but not catastrophic failure. Normally, the part holds its shape at these temperatures - it is designed for operation for long periods of time under those conditions. The business group is worried because they don't know if the failure is an isolated one or indicative of the entire lot. They need answers, and quickly. The first step for the analyst, and this is usually the most difficult, is to interview the client. It is at this point that we must obtain as much information as possible about the sample: what is the recipe, when was the sample made, is this the first time this type of failure has manifested itself, what kind of pre-use testing is done, are there any controls or references, etc.? During this interview, we also have to think on the fly about what kinds of testing would give us the most useful information for the least amount of time and money, so that at the end, we can reassure the client that he or she has come to the right person to solve the problem.

We are fortunate enough to have both a reference and a control: the reference is a pristine, unused part from a different lot, and the control is a part that has been in use and has not failed. From those samples, we can use the first step in the microscopy hierarchy - a visual exam of the shape, dimension, color and condition. How does the control part deform during use? Does the failed part appear or feel different? If so, how? Now we have to classify the type of deformation; once that is done, we have to determine what factors can lead to that condition. Are the elastomer/polymer properties appropriate for the usage? What amount and kind of fillers or reinforcing agents are recommended for a compound chosen for that specific service? How does filler dispersion affect the properties of the compound? What kind of cure is the best for that specific blend of polymers? What external agents (lubes, cleaning agents, heat, etc.) are present that could break down the part? Just in those five questions, we should see that microscopy and microanalysis might not provide all the answers. We can assess carbon black dispersion using optical microscopy examination of sledge microtome sections. Filler dispersion might be done the same way, but would be more accurately accomplished using EDS mapping. Filler levels can be assessed using semi-quantitative EDS comparison among the failed sample, reference and control. Dimension and shape differences that 
were noted in the visual exam can be more accurately measured with a stereomicroscope or in the SEM. We can look for mechanical damage in the form of abrasion, cracks, or cuts using the stereo. Even cure properties among the three can be compared optically by observing timed solvent swelling with phase contrast optical microscopy. Good quick information, yes, but it only leads us to reasons for failure; it does not confirm them or establish quantitative measures to compare to compound and engineering specifications. Infrared analysis is necessary for the elastomer/polymer ID, as well as some of the additives. Nuclear magnetic resonance (NMR) may be needed to determine the elastomer structure. Thermogravimetric analysis (TGA) can tell us the percent composition of the compound with respect to polymers, volatile material, carbon black and ash. Differential scanning calorimetry (DSC) would be an excellent choice to determine if the glass transition temperatures differed among the three samples. If the part were used in a dynamic situation, thermomechanical and dynamic mechanical analyses (TMA and DMA) would yield even better temperature-dependent data than the DSC. If the cure properties appeared different from the solvent swelling method or actual Shore hardness measurements, direct compound mass spectrometry (MS) would give us a fingerprint of the cure package fragments.

OK, now we're talking about many more tests than the client wanted, or what's really important, much more money and time than the client was expecting. This situation begs for the analyst to have an analysis map to navigate the problem logically, and determine what methods will give the most valuable information. The analysis map serves to reassure the client that he or she will not be spending more money or time than is necessary on a shotgun approach that might not provide the reason for the failure. Finally, the map gives us a logical pattern in which to fit the analytical data and arrive at reasonable conclusions about what went wrong and how other parts can be most effectively assessed. This presentation will follow a product problem through the moment it "walks in the door" until a full analysis is complete and related to the client. 